\title{
25 Research Square \\ Risk Factors for Early Seizure After \\ Revascularization in Patients With Moyamoya \\ Disease
}

Hongchuan Niu

Peking University International Hospital

Cunxin Tan

Peking University International Hospital

Kehan Jin

Peking University International Hospital

Ran Duan

Peking University International Hospital

Guangchao Shi

Peking University International Hospital

Rong Wang ( $\nabla$ Ronger090614@ccmu.edu.cn )

Peking University International Hospital

Research

Keywords: Moyamoya disease, Revascularization, Seizure.

Posted Date: September 7th, 2021

DOl: https://doi.org/10.21203/rs.3.rs-789530/v1

License: (9) (i) This work is licensed under a Creative Commons Attribution 4.0 International License.

Read Full License 


\section{Abstract \\ Background}

To investigate the risk factors of early seizure after revascularization in patients with moyamoya disease (MMD).

\section{Methods}

A total of 298 patients with MMD diagnosed in our hospital from 2015 to 2018 were analyzed retrospectively. We summarized the characteristics of seizure after revascularization in patients with MMD and analyzed the predictors of early postoperative seizure.

\section{Results}

We identified 15 patients with MMD who developed seizures within 1 week after revascularization. According to logistic regression analysis, age (OR:1.04, 95\% $\mathrm{Cl} 0.998-1.086 ; \mathrm{P}=0.060)$, and infarct side (OR:1.92, 95\% Cl 0.856-4.290; $\mathrm{P}=0.113$ ) were not significantly associated with incident early seizure. Postoperative infarction (OR:12.89, 95\% Cl 4.198-39.525; $\mathrm{P}=0.000)$ and preoperative cerebral infarction (OR:4.08, 95\% Cl 1.267-13.119; $\mathrm{P}=0.018$ ) were confirmed as risk factors for early seizure.

\section{Conclusions}

We believe that history of preoperative infarction and new infarction are independent risk factors of early seizure in patients with MMD after revascularization.

\section{Background}

Moyamoya disease is a type of cerebrovascular disease with unknown etiology, which is characterized by chronic progressive stenosis or occlusion of the ends of bilateral internal carotid arteries, anterior cerebral arteries, and the beginning of middle cerebral arteries, and is secondary to the formation of abnormal vascular network in the skull base. It was first reported by Japanese scholars Suzuki $\mathrm{J}$ and Takaku in 1969[1]. Both direct and indirect revascularizations or the combination of these two types result in seizure. There are only a few studies on seizure after MMD operation[2-7]. In the present study, we summarize the characteristics of early postoperative seizure in patients with MMD to estimate its risk factors.

\section{Methods}

\section{Patient selection}


We retrospectively analyzed all patients diagnosed with MMD $\ 167.5 \rrbracket I C D-10-C M$ code『in our hospital from 2015 to 2018. The inclusion criteria were as follows: 1. Patients confirmed to have MMD as diagnosed by digital subtraction angiography (DSA) or magnetic resonance angiography (MRA) according to the guidelines of the MMD (Willis circle spontaneous occlusion) Research Committee in 2012; 2. Patients with seizure and abnormal EEG (epileptiform discharge of different degrees) after vascular reconstruction. Patients diagnosed with moyamoya syndrome were excluded.

The study was approved by the research ethics committee of Peking University International Hospital. All patients and their families gave informed consent before participating in the study.

\section{Imaging evaluation}

All patients were scanned by Computed Tomography (CT) or Magnetic Resonance Imaging (MRI) at admission and all patients underwent DSA after admission. According to the angiographic staging of MMD described by Suzuki and Takaku, MMD was classified on the basis of DSA. We used perfusion CT to assess cerebral blood flow (CBF). All the imaging data were independently reviewed by 2 neurologists who were blinded to the patient's information. Any discrepancies were resolved by consensus or by a third party.

\section{Treatment strategy}

The indications of MMD include (1) obvious cerebral ischemia or hemorrhage and (2) recurrent clinical symptoms caused by perfusion injury, including CBF reduction and insufficient reserve. The common operation methods are direct bypass and indirect bypass. Indirect revascularization includes brain dura temporal artery application (EDAs) and burr holes. Direct revascularization included end-to-side anastomosis of the branches of the superficial temporal artery (STA) and the cortical branches of the middle cerebral artery. Combined revascularization (indirect revascularization and direct revascularization on the same side) was analyzed as direct revascularization. For direct bypass, we chose the anterior branch of STA as the donor and M4 branch of the middle cerebral artery as the recipient artery. After craniotomy of small bone flap (3-5 cm in diameter) for the lateral fissure, we performed end-to-side anastomosis of donor and recipient arteries. Indocyanine green angiography confirmed the patency of the grafts. For encephalo-duro-arterio-synangiosis (EDAS), STA was exposed and then sutured to the brain surface. For multiple burr holes, 5-15 holes were placed in the hypoperfusion brain area, and the dura was simultaneously cut and separated at the same time. In our institute, we mainly perform direct vascular reconstruction in all patients, unless the donor or recipient vessels are too small to be anastomosed. In one operation, we performed only unilateral revascularization. For the choice of operative side, symptomatic side or hypoperfusion side is the priority side of revascularization. All patients received antiepileptic drugs after revascularization.

\section{Evaluation of clinical results}


According to "Jennett WB. Early traumatic seizure. Definition and identity. Lancet. 1969;1(7604):10231025. [8] " and the international league against seizure guidelines, early seizure was defined as seizures occurred within 7 days [9]. We collected early epileptic seizures occurred within 1 week after operation. Electroencephalogram (EEG) confirmed that the symptoms of seizures were recorded. According to the classification of epileptic seizures, the duration and types of seizures were recorded for analysis.

\section{Statistical analysis}

Descriptive summaries were reported as mean \pm standard deviation of consecutive variables and the frequency (percentage) of categorical variables. We used logistic regression to calculate the odds ratios (ORs) of $95 \%$ confidence intervals (Cls) and $\mathrm{P}$ value. All the data were statistically analyzed by correlation analysis, $\chi^{2}$ test, one-way ANOVA, and logistic regression to determine the risk factors that were related to the outcome of adverse seizure. Statistical software was used for the analysis of data.

\section{Results}

From 2015 to 2018, 15 (5.0\%) of 298 patients with MMD who underwent revascularization in Peking University International Hospital developed seizure after revascularization (Table 1). Summary of clinical characteristics of patients with or without seizure is as follows (Table 2): There were 6 males (40\%) and 9 females $(60 \%)$ developed seizure after surgery. The sex ratio of epileptic patients and nonepileptic patients was similar $(P=0.711)$. The data of age were tested for normality. The average age of patients with early postoperative seizure was $43.00 \pm 15.21$ years, while the average age of the nonseizure group was $35.24 \pm 15.14$ years. There was no significant age difference between the two groups $(P=0.054)$. 
Table 1

Clinical data of early Epilepsy after Revascularization in MMD patients

\begin{tabular}{|llllllll|}
\hline No. & gender & age & $\begin{array}{l}\text { Cerebral } \\
\text { infarction } \\
\text { before op }\end{array}$ & Revascularization & $\begin{array}{l}\text { Cerebral } \\
\text { infarction } \\
\text { after op }\end{array}$ & $\begin{array}{l}\text { seizure } \\
\text { onset } \\
\text { after } \\
\text { op(d) }\end{array}$ & $\begin{array}{c}\text { Seizure } \\
\text { types } \\
\text { after op }\end{array}$ \\
\hline 1 & M & 41 & Y & bypass + EDAS & Y & 1 & SPS \\
\hline 2 & F & 51 & Y & bypass + EDAS & N & 5 & GTCS \\
\hline 3 & F & 32 & Y & burr holes & N & 1 & CPS \\
\hline 4 & F & 54 & Y & EDAS & Y & 1 & CPS \\
\hline 5 & M & 50 & Y & bypass + EDAS & N & 3 & GTCS \\
\hline 6 & F & 32 & N & bypass + EDAS & N & 5 & GTCS \\
\hline 7 & M & 53 & Y & bypass + EDAS & N & 1 & SPS \\
\hline 8 & F & 45 & Y & EDAS & Y & 1 & SPS \\
\hline 9 & F & 14 & Y & EDAS & Y & 1 & SPS \\
\hline 10 & M & 53 & Y & bypass + EDAS & Y & 1 & SPS \\
\hline 11 & M & 57 & Y & bypass + EDAS & N & 6 & SPS \\
\hline 12 & F & 45 & N & EDAS & Y & 6 & SPS \\
\hline 13 & F & 55 & N & bypass + EDAS & Y & 1 & CPS \\
\hline 14 & F & 55 & Y & bypass + EDAS & N & 1 & SPS \\
\hline 15 & M & 8 & N & EDAS & N & 6 & SPS \\
\hline M: male; F: female; Y: yes; N: no; op: operation; EDAS: encephalo-duro-arterio-synangiosis; SPS: \\
simple partial seizures; CPS: complex partial seizures; GTCS: generalized tonic-clonic seizures.
\end{tabular}


Table 2

Clinical characteristics of patients with EP and without EP after operation

\begin{tabular}{|c|c|c|c|}
\hline & $\begin{array}{l}\text { patient with EP after } \\
\text { operation }\end{array}$ & $\begin{array}{l}\text { patient without EP after } \\
\text { operation }\end{array}$ & $\mathbf{P}$ \\
\hline number of patient & 15 & 283 & \\
\hline age(years) & $43.00 \pm 15.208$ & $35.24 \pm 15.140$ & 0.054 \\
\hline male & 6 & 127 & 0.711 \\
\hline infraction before op & 10 & 102 & 0.011 \\
\hline infraction side before op & & & 0.042 \\
\hline left & 4 & 51 & \\
\hline right & 1 & 33 & \\
\hline both & 5 & 18 & \\
\hline hemorrhage before op & 0 & 68 & 0.031 \\
\hline EP before op & 3 & 23 & 0.806 \\
\hline history of EP(months) & $4.52 \pm 36.814$ & $3.20 \pm 12.394$ & 0.89 \\
\hline SE before op & 1 & 4 & 0.025 \\
\hline surgical modalities & & & 0.907 \\
\hline bypass & 0 & 1 & \\
\hline EDAS & 5 & 77 & \\
\hline bypass + EDAS & 9 & 187 & \\
\hline burr holes & 1 & 8 & \\
\hline EDAS + burr holes & 0 & 9 & \\
\hline bypass + EDAS + burr holes & 0 & 1 & \\
\hline $\begin{array}{l}\text { Postoperative hyperperfusion } \\
\text { syndrome }\end{array}$ & 0 & 5 & 0.604 \\
\hline infraction after op & 7 & 18 & 0 \\
\hline
\end{tabular}

There were 112 patients with cerebral infarction before operation; 10 of them had epileptic seizures after operation, and the results were significantly different $(P=0.011)$. Four of the 10 patients with cerebral infarction were left, one was right, and five were bilateral. According to the $\chi 2$ test, bilateral cerebral 
infarction was observed before operation, and the incidence of early epileptic seizures was high $(P=$ 0.042). Among 68 patients with MMD, none of them had early seizure after operation. According to the correlation analysis, a negative correlation was observed preoperative cerebral hemorrhage and postoperative early seizure $(P=0.031)$. There were 26 patients with previous epileptic history before operation, but only 3 patients had epileptic seizure after operation, and there was no significant difference $(P=0.806)$. The average medical history of 3 patients was $4.52 \pm 36.814$ months, the average medical history of 23 patients with early postoperative seizure was $3.20 \pm 12.394$ months, and the difference was not statistically significant $(P=0.890)$. Five patients had status epileptecus before operation, and one of these patients had seizure postoperatively $(P=0.025)$. In the seizure group, 9 patients $(60 \%)$ underwent direct reconstruction, 5 patients (33.3\%) underwent EDAs, and 1 patient (6.7\%) underwent burr holes. There was no significant difference in the choice of surgical modifications. After operation, 5 patients had hyperperfusion syndrome, and none of them had early seizure. There were 25 patients with new cerebral infarction after operation, and 7 (28.0\%) of them had early seizure. The statistical analysis revealed a positive correlation between new cerebral infarction and early seizure $(P=0.000)$

The analysis of risk factors of early seizure postoperatively is shown in Table 3. Logistic regression revealed that age (OR:1.04, 95\% $\mathrm{Cl} 0.998-1.086 ; \mathrm{P}=0.060)$ and infarct side (OR:1.91, 95\% $\mathrm{Cl} 0.856-$ 4.290; $P=0.113$ ) were not significantly associated with incident early seizure. Postoperative infarction (OR:12.88, 95\% Cl 4.198-39.525; $\mathrm{P}=0.000)$ and preoperative cerebral infarction (OR:4.07, 95\% Cl 1.26713.119; $P=0.018$ ) were identified as risk factors for early seizure.

Table 3

Variables of factors associated with early epilepsy after revascularization analyzed by logistic regression

\begin{tabular}{|c|c|c|c|c|c|c|c|c|}
\hline \multirow[t]{2}{*}{ Variable } & \multirow[t]{2}{*}{ B } & \multirow[t]{2}{*}{ S.E, } & \multirow[t]{2}{*}{ Wald } & \multirow[t]{2}{*}{$\mathbf{v}$} & \multirow[t]{2}{*}{$\mathbf{P}$} & \multirow[t]{2}{*}{$\operatorname{Exp}(B)$} & \multicolumn{2}{|c|}{$95 \% \mathrm{Cl} 95 \% \mathrm{Cl}$} \\
\hline & & & & & & & $\begin{array}{l}\text { Lower } \\
\text { limit }\end{array}$ & $\begin{array}{l}\text { Upper } \\
\text { limit }\end{array}$ \\
\hline Constant & -4.527 & 0.955 & 22.481 & 1 & 0.000 & 0.011 & & \\
\hline Age & 0.040 & 0.021 & 3.531 & 1 & 0.060 & 1.041 & 0.998 & 1.086 \\
\hline Prior_infarction_side & 0.651 & 0.411 & 2.505 & 1 & 0.113 & 1.917 & 0.856 & 4.290 \\
\hline $\begin{array}{l}\text { Postoperative } \\
\text { infarction }\end{array}$ & 2.556 & 0.572 & 19.965 & 1 & 0.000 & 12.882 & 4.198 & 39.525 \\
\hline Prior_infarction & 1.405 & 0.596 & 5.554 & 1 & 0.018 & 4.077 & 1.267 & 13.119 \\
\hline EP & -0.260 & 1.057 & 0.061 & 1 & 0.805 & 0.771 & 0.097 & 6.117 \\
\hline
\end{tabular}

\section{Discussion}


In this study, the average ratio of male to female was 1:1.22 (male 134: female 164), with 57 children and 241 adults, and the incidence of preoperative seizure was approximately $8.72 \%$. This is basically consistent with the results of previous studies. For the 941 cases of MMD in Japan, the female: male ratio was 1.98:1. The two peaks of age of onset were 5-9 years and about 40 years. The incidence of seizure was 4\%[10]. For children with MMD, Ma reported in 2017 that the proportion of men and women with seizure was approximately $1: 0.87$, and there was no difference in the sex ratio, with an incidence of $18.1 \%[11]$.

Patients with MMD report transient ischemic attack (TIA) before operation, but it is difficult to distinguish from some epileptic attacks in the form of expression. It was found that the occurrence of cerebral infarction before operation and new infarction after operation were the risk factors of early seizure in patients with MMD after revascularization. There are two mechanisms that may lead to early and late epileptic seizures after neurosurgery. One mechanism is the extravasation of blood vessels. Iron released from hemoglobin reacts with hydrogen peroxide in the surrounding tissues to produce free radicals, which may lead to early epileptic seizures and the formation of epileptic foci. Hydrogen peroxide reduces the inhibition of $y$-aminobutyric acid (GABA)-mediated cortical and thalamic neurons. Free radicals can promote the excitability of thalamic cortical circuits by changing the neurotransmitter mediated by $\beta$ aminobutyric acid. Repeated exposure to free radicals will lead to the formation of epileptic foci. Another mechanism may be through disorder of ion balance in the cell membrane caused by ischemia or hypoxia. The decrease of high energy reserve, such as adenosine triphosphate under the condition of ischemia or hypoxia, further leads to the disorder of ion balance on the cell membrane; this is because the ratio of intracellular and extracellular $\mathrm{K}+$ concentration decreases, whereas the ratio of sodium ion concentration increases. This imbalance reduces the hyperpolarization of neurons that may cause transient or early seizures. The imbalance of sodium ions is mainly mediated by the outflow of Ca2+-independent glutamate during ischemia[12].

Choi Ji et al found that 4/7 of the patients with MMD had seizure with hypoperfusion, [13] and stroke and hypoperfusion would lead to cerebrovascular reactive disorder, followed by hypoxia of brain tissue and seizure. Blood flow reconstruction can improve the perfusion of brain tissue and prevent seizure[13]. According to Mikami et al, moyamoya seizure is a typical type of seizure after stroke. However, in a previous study, the severity of vascular stroke shown by MRA was not related to seizure[14]. The prognosis of MMD epileptics with abnormal CT findings was poor[15]. Ma et al suggested that preoperative cerebral infarction could not predict the recurrence of seizure[11].

Ma et al. found that the duration of epileptic attack before operation was an independent risk factor for seizure recurrence after revascularization of children with MMD[11], and revascularization should be performed immediately after the diagnosis of seizure. In our study, there was no correlation between preoperative seizure history and postoperative seizures. In this group of patients, we found that the preoperative status of seizure in patients with MMD may be related to the early postoperative seizures as shown by one-way ANOVA. A few other studies have reported on seizure caused by MMD, but in other studies, it was related to cerebrovascular diseases. According to Englot et al, the seizure time was less 
than 1 year, which is a predictor of seizure in patient with cavernous malformation[16]. According to Falero and León, the short seizure time of arteriovenous malformation (AVM) was statistically significant with class I after operation[17]. Liu et al believes that the long duration of AVM indicates poor prognosis[18].

In this group of cases, the seizure forms of patients with seizure before operation include simple partial seizures (SPS), complex partial seizures (CPS), and generalized tonic-clonic seizures (GTCS). Among the 23 epileptic patients before operation, 12 had SPS attack and 6 had CPS attack. Some of them had high incidence, which may be related to focal infarction or ischemic focus before operation. However, we found that patients with persistent state of seizure before operation are more likely to have early seizure after undergoing revascularization. Few researchers examined the relationship between seizure form and seizure after operation for MMD, while in other cerebrovascular disease studies, seizure form is related to the prognosis of patients; moreover, there are also contrary findings. GTCS attack in cavernous malformation (CM) patients indicates poor prognosis[19-22]. According to Falero and León, GTCS attack in AVM indicates a good prognosis[17]. In patients with MMD, there was no correlation between attack pattern and prognosis.

In the present study, we did not find that the choice of operation mode was related to the early postoperative seizure. For patients with ischemic MMD and high risk of preoperative infarction, we chose EDAS operation with shorter operation time to reduce the risk of anesthesia and operation. If there are receptor vessels with appropriate thickness and flow, the bypass operation is more feasible. Therefore, there is a certain bias in the selection of operation method, which is often compared with decisionmaking. According to Choi et al, EDAS is safe and effective to prevent postoperative seizure[13]. Ma 2017 found no difference between the two surgical methods (direct / indirect) in the prevention of postoperative seizure[11]. There are still many concerns associated with the specific operation. In this group, the blood supply reconstruction operation mode involves cutting the skin craniotomy and milling the bone flap along the superficial temporal artery. Some units use the frontotemporal craniotomy and mill the bone flap. However, it is unclear (1) whether there is any influence of the size of bone window exposing the brain tissue area on the postoperative seizure, (2) whether there is stimulation of the connective tissue around the superficial temporal artery, (3) whether there is traction and contusion of brain tissue during the operation, and (4) whether there is subarachnoid hemorrhage during the operation. This information may have an impact on the postoperative seizure, but no detailed data can be used for in-depth statistics.

Hyperperfusion of brain tissue after STA-MCA bypass can cause transient neurological dysfunction, including postoperative seizure[23]. However, there are similar biological mechanisms between postoperative seizure and postoperative hyperperfusion syndrome[12, 24]. Postoperative high perfusion syndrome can cause headache, eye and facial pain, epileptic seizure, and focal neurological dysfunction secondary to brain edema. The pathological mechanism may be the impairment of cerebrovascular selfregulation function, which makes it difficult to regulate the blood flow from the superficial temporal artery to the middle cerebral artery after bypass surgery. In addition, oxygen free radicals produced during 
reperfusion after revascularization may damage cerebral vessels, leading to the occurrence or aggravation of high perfusion. The intrinsic response of some revascularization, such as the increase of vascular permeability, may be related to high perfusion after revascularization in MMD patients. Longterm cortical ischemia can induce the overexpression of angiogenic factors and extracellular matrix proteins, thus promoting the formation of new blood vessels and increasing the permeability of blood vessels[25].

According to the new National Institute for Health and Care Excellence guidelines, carbamazepine and lamotrigine are the first-choice drugs for focal seizure[26]. Most of the cases of moyamoya occur in young women. For the long-term control of moyamoya seizure, lamotrigine and levetiracetam can be used as the first-choice drugs in consideration of tolerance and family burden[14].

The present study has several limitations: first, although the total number of patients with MMD included in this study is quite large, the number of seizure cases after operation is insufficient. To confirm our conclusion, a larger sample size and randomized design of future research are needed. Second, it is impossible to collect more detailed information about the operation details and techniques. Perhaps more detailed observation indicators should be taken in the future research design, in order to obtain more detailed conclusions.

\section{Conclusions}

Our study showed that the history of pre-operative infarction and new postoperative infarction are independent risk factors for early postoperative seizure in patients with MMD.

\section{Abbreviations}

MMD: Moyamoya disease; ICA: Internal carotid artery; MCA: Middle cerebral artery; ACA: Anterior cerebral artery; TIA: Transient ischemic attack; EDAS: Encephaloduroarteriosynangiosis; EMS:

Encephalomyosynangiosis; STA-MCA: Superficial temporal artery-middle cerebral artery. DSA: Digital subtraction angiography

\section{Declarations}

\section{Acknowledgements}

Not applicable

\section{Authors' contributions}

HN substantial contributions to the conception or design of the work or the acquisition, analysis, or interpretation of the data for the work. CT conducted the statistical analysis; and was the equal contributor in writing this manuscript. $\mathrm{KJ}$ was responsible for proofreading the collected studies and 
rechecking the data. RD collected the patients' information. GS was responsible for ensuring that the final statistical analysis of the data was accurate and reliable.RW reviewed and modified this manuscript, and was the major corresponding author of this manuscript. All authors read and approved the final manuscript.

\section{Funding}

Not applicable

\section{Availability of data and materials}

All data generated or analyzed during this study are included in these published articles.

\section{Ethics approval and consent to participate}

Not applicable

\section{Consent for publication}

Not applicable

\section{Competing interests}

The authors declare that they have no competing interests.

\section{Author details}

1Peking University International Hospital, Department of Neurosurgery, Peking University, Beijing, China. 2 Peking University International Hospital, Department of Neurology, Peking University, Beijing, China. 3Beijing Tiantan Hospital, Department of Neurosurgery, Capital Medical University, Beijing, China.

\section{References}

1. Jiro Suzuki;Akira Takaku. Cerebrovascular "Moyamoya" Disease.Disease Showing Abnormal Net-Like Vessels in Base of Brain. Arch Neurol. 1969;20(3):288-99.

2. Morimoto M, Iwama T. NHAKKH. Efficacy of Direct Revascularization in Adult Moyamoya Disease: Haemodynamic Evaluation by Positron Emission Tomography. Acta Neurochir (Wien). 1999;141:377-84.

3. De Reuck J, Goethals M, Vonck K, Van Maele G. Clinical predictors of late-onset seizures and epilepsy in patients with cerebrovascular disease. Eur Neurol. 2005;54(2):68-72.

4. Hayashi K, Horie N, Suyama K, Nagata I. Clinical features and long-term follow-up of quasimoyamoya disease in children. Pediatr Neurosurg. 2011;47(1):15-21. 
5. Morais NMM, Ranzan J, Riesgo RS. Predictors of Epilepsy in Children With Cerebrovascular Disease. J Child Neurol. 2012;28(11):1387-91.

6. Gibson LM, Hanby MF, Al-Bachari SM, Parkes LM, Allan SM, Emsley HC. Late-onset epilepsy and occult cerebrovascular disease. J Cereb Blood Flow Metab. 2014;34(4):564-70.

7. Hirano T, Enatsu R, lihoshi S, Mikami T, Honma T, Ohnishi H, Mikuni N. Effects of Hemosiderosis on Epilepsy Following Subarachnoid Hemorrhage. Neurol Med Chir (Tokyo). 2019;59(1):27-32.

8. Jennett WB. Early traumatic epilepsy. Definition and identity. Lancet. 1969;1(7604):1023-5.

9. Commission on Epidemiology and Prognosis ILAE. Guidelines for Epidemiologic Studies on Epilepsy. Epilepsia. 1993;34(4):592-6.

10. HOSHINO H, IZAWA Y, SUZUKI N, Diseas RCoM. Epidemiological Features of Moyamoya Disease in Japan. Neurol Med Chir (Tokyo). 2012;52:295-8.

11. Ma Y, Zhao M, Zhang Q, Liu X, Zhang D, Wang S, Zhang Y, Li M, Zhao J. Risk Factors for Epilepsy Recurrence after Revascularization in Pediatric Patients with Moyamoya Disease. J Stroke Cerebrovasc Dis. 2018;27(3):740-6.

12. MANAKA S, ISHIJIMA B. MAYANAGI Y. Postoperative Seizures: Epidemiology, Pathology, and Prophylaxis. Neurol Med Chir (Tokyo). 2003;43:589-600.

13. Choi JI, Ha SK, Lim DJ, Kim SD. Differential clinical outcomes following encephaloduroarteriosynangiosis in pediatric moyamoya disease presenting with epilepsy or ischemia. Childs Nerv Syst. 2015;31(5):713-20.

14. Mikami T, Ochi S, Houkin K, Akiyama Y, Wanibuchi M, Mikuni N. Predictive factors for epilepsy in moyamoya disease. J Stroke Cerebrovasc Dis. 2015;24(1):17-23.

15. NAKASE H, OHNISHI H, TOUHO H, MIYAMOTO S, WATABE Y, ITOH T, YAMADA K, KARASAWA J, SAKAMOTO T, KUREHARA K. SHIMIZU K. Long-term Follow-up Study of "Epileptic Type" Moyamoya Disease in Children. Neurol Med Chir (Tokyo). 1993; 33.

16. Englot DJ, Han SJ, Lawton MT, Chang EF. Predictors of seizure freedom in the surgical treatment of supratentorial cavernous malformations. J Neurosurg. 2011;115(6):1169-74.

17. Falero RAP, León OH. Re: results of multimodality treatment for 141 patients with brain arteriovenous malformations and seizures: factors associated with seizure incidence and seizure outcomes. Neurosurgery. 2005;56(3):E629.

18. Liu S, Chen HX, Mao Q, You C, Xu JG. Factors associated with seizure occurrence and long-term seizure control in pediatric brain arteriovenous malformation: a retrospective analysis of 89 patients. BMC Neurol. 2015;15:155.

19. Janszky J, Janszky I, Schulz R, Hoppe M, Behne F, Pannek HW, Ebner A. Temporal lobe epilepsy with hippocampal sclerosis: predictors for long-term surgical outcome. Brain. 2005;128(Pt 2):395-404.

20. Mclntosh AM, Kalnins RM, Mitchell LA, Fabinyi GC, Briellmann RS, Berkovic SF. Temporal lobectomy: long-term seizure outcome, late recurrence and risks for seizure recurrence. Brain. 2004;127(Pt 9):2018-30. 
21. Jeong S-W, Lee SK, Hong K-S, Kim K-K, Chung C-K, Kim H. Prognostic factors for the surgery for mesial temporal lobe epilepsy: Iongitudinal analysis. Epilepsia. 2005;46:1273-9.

22. Cendes F, Andermann F, Gloor P, Gambardella A, Lopes-Cendes I, Watson C, Evans A, Carpenter S, Olivier A. Relationship between atrophy of the amygdala and ictal fear in temporal lobe epilepsy. Brain. 1994;117:739-46.

23. Fujimura M, Shimizu H, Inoue T, Mugikura S, Saito A, Tominaga T. Significance of focal cerebral hyperperfusion as a cause of transient neurologic deterioration after extracranial-intracranial bypass for moyamoya disease: comparative study with non-moyamoya patients using $\mathrm{N}$-isopropyl-p[(123)]]iodoamphetamine single-photon emission computed tomography. Neurosurgery. 2011;68(4):957-64. discussion 964 - 955.

24. van Mook WNKA, Rennenberg RJMW, Schurink GW, van Oostenbrugge RJ, Mess WH, Hofman PAM, de Leeuw PW. Cerebral hyperperfusion syndrome. Lancet Neurol. 2005;4(12):877-88.

25. Zhao WG, Luo Q, Jia JB, Yu JL. Cerebral hyperperfusion syndrome after revascularization surgery in patients with moyamoya disease. Br J Neurosurg. 2013;27(3):321-5.

26. Nunes VD, Sawyer L, Neilson J, Sarri G, Cross JH. Diagnosis and management of the epilepsies in adults and children: summary of updated NICE guidance. BMJ. 2012;344:e281. 